\title{
FINE NEEDLE ASPIRATION CYTOLOGY IN DIAGNOSIS OF HODGKIN'S LYMPHOMA
}

\section{PARIKH U.R. ${ }^{*}$, GOSWAMI H.M. ${ }^{1}$, MEHTA N.P. ${ }^{1}$, MEHTA R. ${ }^{1}$, YADAV K.S. ${ }^{2}$, SHAH P.K. ${ }^{3}$ AND SUDHAMANI S. ${ }^{2}$}

1Department of Pathology, B.J. Medical College, Asarwa, Ahmedabad-380016, Gujarat, India.

2Padmashree Dr. D. Y. Patil Medical College \& Hospital, Navi Mumbai-400706, MS, India.

3Department of Microbiology, B.J. Medical College, Asarwa, Ahmedabad-380016, Gujarat, India.

${ }^{\star}$ Corresponding Author: Email- urviparikh76@gmail.com

Received: July 14, 2012; Accepted: July 24, 2012

\section{Abstract-}

Introduction- Hodgkin's disease primarily involves lymphoreticular system with characteristic tumor cells and infiltration of reactive lymphocytes, eosinophils and plasma cells. It is said that FNAC has greater diagnostic accuracy in Hodgkin's lymphoma than non Hodgkin's lymphoma.

Aim- The present study is undertaken to determine the incidence and age wise distribution of Hodgkin's disease in our tertiary care hospital.

Material and Method- A retrospective study of 1194 consecutive patients with lymphadenopathy was conducted over a period of eighteen months; between July, 2010 to December, 2011 in the department of pathology this hospital. Aspiration was done after taking detailed clinical history and physical examination of the patient. FNAC results were compared with final histopathological diagnosis.

Results- FNA results were classified as inadequate material, non-neoplastic lesions and neoplastic lesions which include metastatic carcinoma, Hodgkin's lymphoma and non Hodgkin's lymphoma. Out of all the studied lymph nodes (1194) during above mentioned period, 17 patients showed cytomorphology of Hodgkin's lymphoma (1.42\%). Age distribution was between 5 years to 70 years. The peak incidence was noted in first decade of life. Hodgkin's lymphoma also showed male predominance with male: female ratio of 7.5:1. The initial affected site most commonly seen was cervical lymph node ( 11 cases). The most frequent initial chief complaint was a palpable mass.

Conclusion- FNAC is safe and inexpensive, outdoor patient procedure with a high diagnostic yield, accuracy, sensitivity and specificity.

Key words- Fine Needle Aspiration Cytology (FNAC), Hodgkin's lymphoma (HL), Reed Sternberg cell (R-S Cell).

Citation: Parikh U.R., et al. (2012) Fine Needle Aspiration Cytology in Diagnosis of Hodgkin's Lymphoma. International Journal of Molecular Biology, ISSN: 0976-0482 \& E-ISSN: 0976-0490, Volume 3, Issue 3, pp.-58-61.

Copyright: Copyright@2012 Parikh U.R., et al. This is an open-access article distributed under the terms of the Creative Commons Attribution License, which permits unrestricted use, distribution and reproduction in any medium, provided the original author and source are credited.

\section{Introduction}

Hodgkin lymphoma is a cancer that begins in cells of the immune system. The immune system fights infections and other diseases. Hodgkin's lymphoma was first described by Thomas Hodgkin in 1832. Many studies have been done concerning its etiology, classification, treatment and prognosis [1]. Hodgkin's lymphoma is a malignant lymphoproliferative disorder with characteristic features regarding epidemiology and histopathology [2]. Although much more progress had been made in classification, treatment and prognosis of this disease; the etiology and the origin of the tumor cell are in much controversy. With respect to etiology, association with Epstein-Barr virus has been suggested [1]. The distribution of incidence, age and histological type is different between advanced, developing and underdeveloped countries [1,2]. In spite of Hodgkin's lymphoma accounting for one third of all false negative diagnosis among lymphoma, FNAC is said to have a higher diagnostic accuracy in Hodgkin's lymphoma (91.8\%) as compared to Non Hodgkin's lymphoma [3]. The purpose of this study is to note the clinical manifestation and age wise incidence of Hodgkin's lymphoma in our tertiary care hospital. 


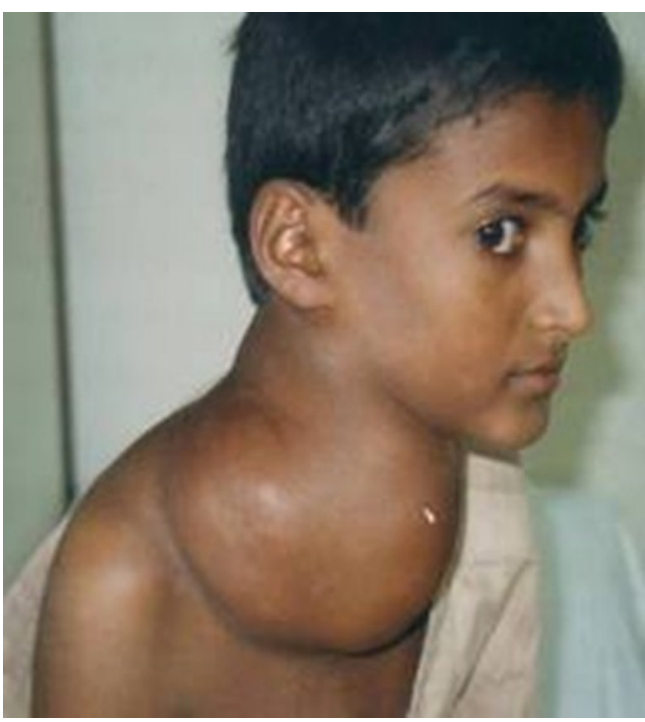

Fig. 1a-

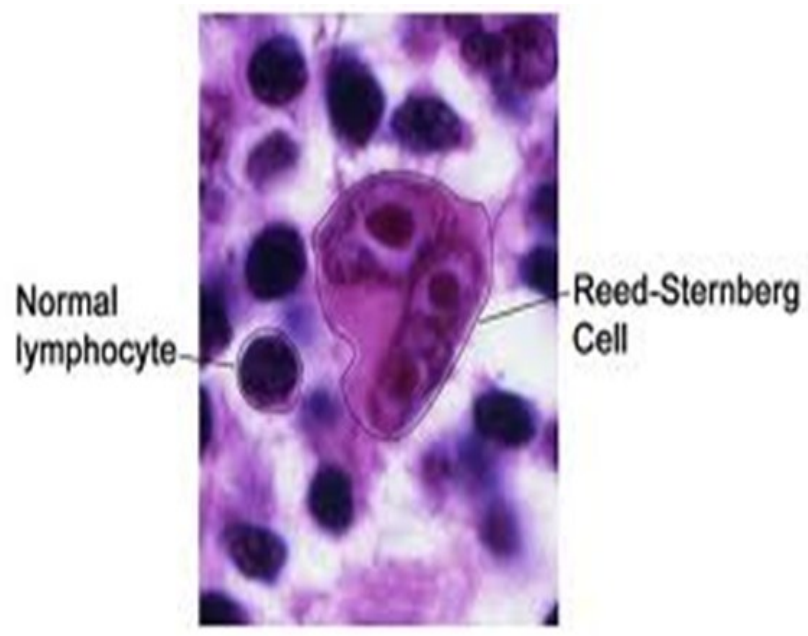

Fig. 1b-

Hodgkin's disease (sometimes referred to as Hodgkin's lymphoma) and non-Hodgkin's lymphoma are cancers that originate in a type of white blood cell known as a lymphocyte, an important component of the body's immune system. Both of these malignancies may cause similar symptoms, but the conditions themselves are different. The distinction between Hodgkin's disease and nonHodgkin's lymphoma is made upon examination of the cancerous material (from a biopsy or aspiration of the tumor tissue). The type of abnormal cells identified in the sample determines whether a lymphoma is classified as Hodgkin's disease or non-Hodgkin's lymphoma.

\section{Risk Factors}

Clinician's wonders why one person develops Hodgkin lymphoma and another does not. But research shows that certain risk factors increase the chance that a person will develop this disease.

The risk factors for Hodgkin lymphoma include the following:

- Certain Viruses: Patient infected with the Epstein-Barr virus (EBV) or the human immunodeficiency virus (HIV) may have high risk of developing Hodgkin lymphoma. However, lympho- ma is not contagious.

- Weak Immune System: The risk of developing Hodgkin lymphoma may be increased by having a weak immune system may be because of an inherited condition or certain immunosuppressant drugs used after an organ transplant.

- Age: Hodgkin lymphoma is most common among teens and adults aged 15 to 35 years and adults aged 55 years and older.

- Family History: Family members, especially brothers and sisters, of a person with Hodgkin lymphoma or other lymphomas may have an increased risk of developing this disease.

Having one or more risk factors does not mean that a person will develop Hodgkin lymphoma. Most people who have risk factors never develop cancer.

\section{Materials and Methods}

A retrospective study of 1194 patients of lymphadenopathy over a period of eighteen months i.e. from July, 2010 to December, 2011 was carried out to determine the age and sex wise incidence of Hodgkin's lymphoma in our tertiary care hospital, in Pathology department. Each of the 17 cases diagnosed as Hodgkin's lymphoma were reviewed in detail regarding detailed clinical history including age, sex, chief complaint, sites of involvement, clinical stage of patient and physical examination. Ultrasonography examination were carried out as and when required and also to know the clinical stage of the patient. Aspiration was performed by 23-24 gauge needle. The smears were fixed with $95 \%$ ethyl alcohol and stained by May Graunwald Giemsa stain, Haematoxyline and eosin stain and Papanicolau's stain. In all the 17 cases of Hodgkin's lympho$\mathrm{ma}$, the diagnosis is confirmed by histopathological examination. Cytology and Histology specimens were analyzed by two examiners who were unaware of each other's diagnosis.

\section{Results}

Many of the patients presented with lymphadenopathy had been diagnosed and treated at our hospital over a period of 18 months, i.e. from July, 2010 to December, 2011 were taken into consideration. Out of 1194 patient of lymphadenopathy, 17(1.42\%) patients, 15 male and 2 females, had been diagnosed as Hodgkin's lymphoma during their FNAC examination. Table 1 showed age and sex wise distribution of Hodgkin's lymphoma. Our youngest patient was 5 year old male child while the oldest patient was 67 year male. Maximum numbers of patients presented in first decade of life (35.29\%). This suggests that children are affected more frequently than adults. Table 1showsage wise distribution of Hodgkin's lymphoma.

Table 1-

\begin{tabular}{|llllll|}
\hline Sr. No. & Age Group (Years) & Male & Female & Total & Percentage (\%) \\
\hline 1 & $01-10$ & 5 & 1 & 6 & 35.29 \\
2 & $11-20$ & 3 & - & 3 & 17.65 \\
3 & $21-30$ & 2 & 1 & 3 & 17.65 \\
4 & $31-40$ & 1 & - & 1 & 5.88 \\
5 & $41-50$ & 3 & - & 3 & 17.65 \\
6 & $51-60$ & - & - & - & - \\
7 & $61-70$ & 1 & - & 1 & 5.88 \\
& TOTAL & 15 & 2 & 17 & \\
\hline
\end{tabular}




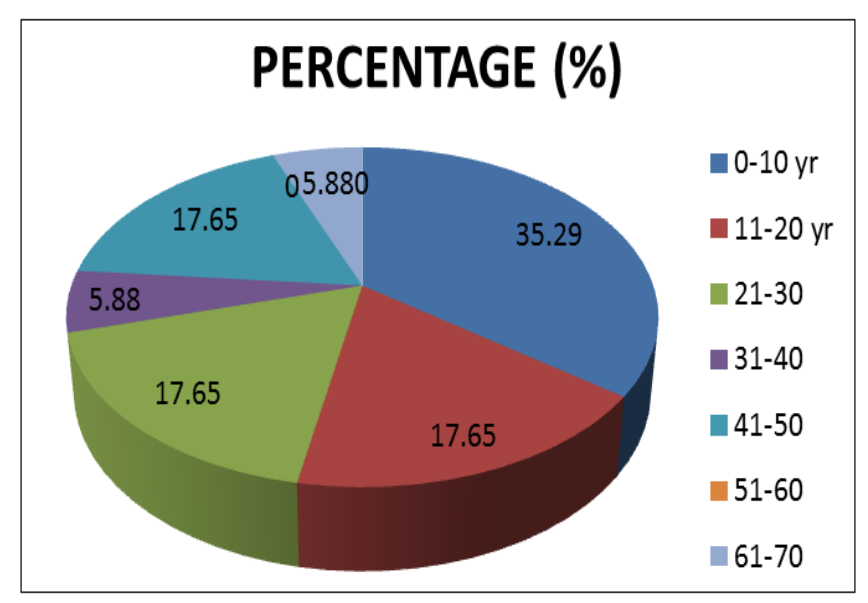

Chart 1- Age wise distribution of Hodgkin's lymphoma

During the present study, 15 male and 2 female patients had been diagnosed as Hodgkin's lymphoma. Hodgkin's lymphoma showed male predominance with male:female ratio of $7.5: 1$. The most common chief complaint was peripheral lymphadenopathy noted in 16 patients during the present study (94.12\%). Table 2 shows Initial FNAC site of Hodgkin's Lymphoma.

Table 2-

\begin{tabular}{|llll|}
\hline Sr. No. Group of Lymph Node & No. of Patients & Percentage (\%) \\
\hline 1 & Cervical lymph node & 11 & 64.71 \\
2 & Cervical and Axillary lymph node & 2 & 11.76 \\
3 & Axillary lymph node & 1 & 5.88 \\
4 & Generalized lymphadenopathy & 2 & 11.76 \\
5 & Abdominal lymphadenopathy & 1 & 5.88 \\
& TOTAL & 17 & $100 \%$ \\
\hline
\end{tabular}

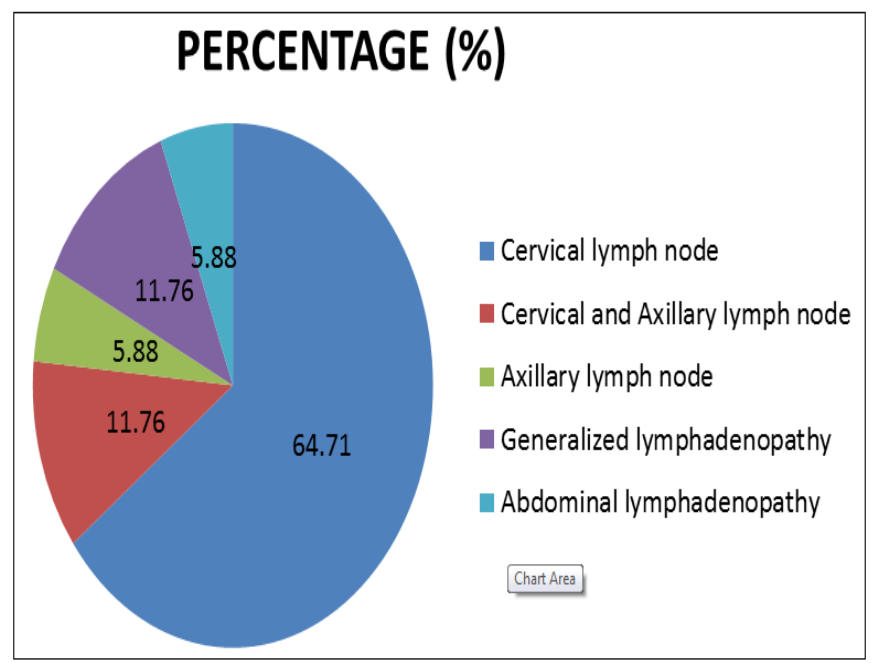

Chart 2- Initial FNAC site of Hodgkin's Lymphoma

The most common group of involvement was cervical group, noted in 11 patients $(64.71 \%)$. The other common complaints were fever, anorexia, generalized weakness and weight loss. One of our patients presented with involvement of abdominal group of lymph node, with absence of peripheral lymphadenopathy. Table 3. Clinical stages of the patients at the time of diagnosis of Hodgkin's Lymphoma.

In that patient, USG guided FNAC was carried out from Paraumbilical group of lymph nodes and diagnosis was made. In all the patients' ultrasonography examination was carried out to confirm the clinical stage.

Table 3-

\begin{tabular}{|llll|}
\hline Sr. No. & Stage of Patient & No. of Patients & Percentage (\%) \\
\hline 1 & Stage I & 10 & 58.82 \\
2 & Stage II & 4 & 23.53 \\
3 & Stage III & 2 & 11.76 \\
4 & Stage IV & 1 & 5.88 \\
& TOTAL & 17 & $100 \%$ \\
\hline
\end{tabular}

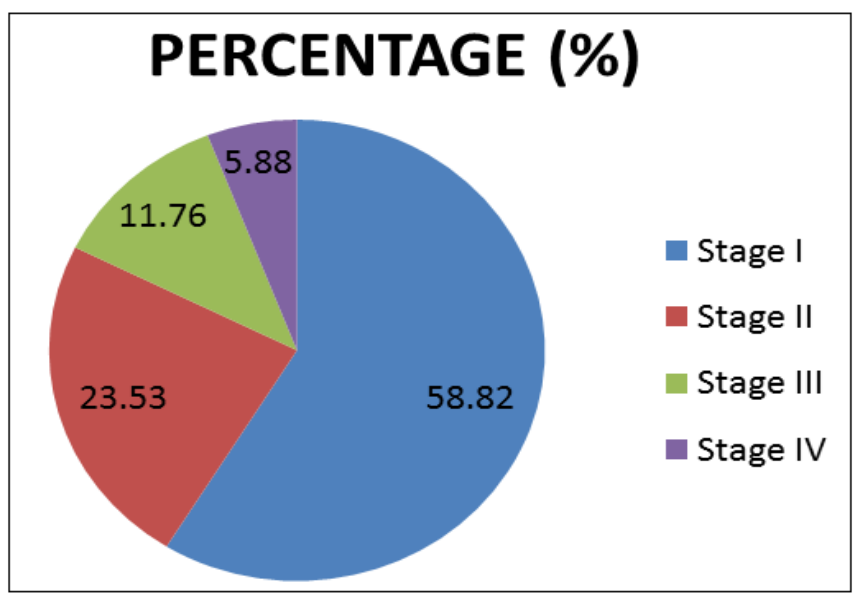

Chart 3-Clinical stages of the patients at the time of diagnosis of Hodgkin's Lymphoma

\section{Discussion}

FNAC is regarded as the gold standard initial investigation in the evaluation of lymph nodes for the diagnosis of lymphomas. The technique is safe, simple and quick with a low complication rate. Several other tests, such as high resolution ultrasonography and FNA biopsy have been used for evaluation of lymphadenopathy before proceeding to surgery. Studies have demonstrated that among all these diagnostic modalities, FNAC is the most accurate, cost effective screening test for rapid diagnosis of lymphadenopathy. In conjunction with immunophenotyping and molecular studies, it has gained acceptance in many centers as a diagnostic tool[4].

During the present study, it is noted that maximum numbers of the patients presented in their first decade of life. This suggests that Hodgkin's lymphoma is more common in children than adults. India is one of the developing countries and here the children are affected more frequently [2]. The similar results were noted by Masko Kusuda, et al [2]. In industrialized countries, this disease shows a bimodal age distribution with peaks in young adults and in elderly, with a predominance of nodular sclerosis subtype. However, in the developing countries, this disease is seen more frequently in children with a predominance of mixed cellularity and lymphocyte depletion subtypes [2].

During the present study, we found that the males are affected more frequently than female; with the male: female ratio of $7.5: 1$. The similar results noted by Masko Kusuda, et al [2].

The initial symptom of Hodgkin's lymphoma is enlargement of peripheral lymph node, noted in 16 out of 17 patients (94.12\%) during the present study. The similar results were noted by Soolm Choi, et 
al, as in his study, 93.10\% patients had peripheral lymphadenopathy [1]. Out of all peripheral groups of lymph nodes, cervical group is involved more commonly, as noted in $60.7 \%$ patients during the present study. The similar results were noted by Soolm Choi, et al [1]. Other than the peripheral lymph nodes; mediastinal lymph node, thymus, tonsil and gastrointestinal tract were also involved. Although, the primary site of involvement in Hodgkin's disease is generally the immune system i.e. the lymphoid organs, but it may metastasize to the gastrointestinal tract, bone marrow or liver [1].

The cornerstone of Cytodiagnosis of Hodgkin's Lymphoma is the finding of classic Reed-Sternberg/ Hodgkin cells in an appropriate polymorphous cellular background [5]. The wide spectrum of cytological features seen in Hodgkin's lymphoma reflects not only the histological diversity of the disease but also overlaps considerably with other reactive and malignant lesions, leading to problems in the diagnosis. In the present study, although classic R-S cells were found in all cases, atypical mononuclear cells with prominent nucleoli apparently outnumbered them and proved to be a better indicator in cytological evaluation [6].

Furthermore, when present, R-S cell need to be differentiated from immunoblasts, which typically are not only smaller than R-S cells but also lack prominent nucleoli and may have plasmacytoid appearance [7].

Another noteworthy problem is that of separating $\mathrm{HL}$ from peripheral T-cell lymphoma (PTCL) that may contain cells which are known to be notorious for mimicking R-S cells. Although this problem has been well addressed in a study by Mathur, et al. and criteria have been proposed to delineate the two, but practical difficulties do persist [8].

Another confounding factor is the presence of an exuberant granulomatous response in association with $\mathrm{HL}$, which may distract the observer from the underlying pathology [9]. During the present study, we noted that one of our patients was previously reported as granulomatous lymphadenitis due to presence of well-formed granuloma. Meticulous search for atypical cells that stand out in a reactive background, along with clinical suspicion may resolve the issue in these cases up to some extent.

In Hodgkin's disease, various immunological defects, especially in cell mediated immunity, cause a disturbance of delayed hypersensitivity, which leads to infections by mycobacterium, histoplasma, Cryptococcus, herpes or toxoplasma [10].

In Hodgkin's disease, both the histological type and the clinical stages are associated with prognosis [11].

\section{Conclusion}

FNA is a very useful tool in the initial diagnosis of $\mathrm{HL}$, but one has to always be aware of its pitfalls. Classic R-S cells are not always abundant. Instead, the presence of atypical mononuclear cells and granuloma together should raise a high index of suspicion for further evaluation. A careful search for these features in a reactive background must be followed. FNA identify the patients who need a biopsy especially in cases with strong clinical suspicion and hence, is a good screening tool.

\section{References}

[1] Soolm Choi, Kyu Rae Kim, Hyeon Joo Jeong, Chan II Park and In Joon Choi (1988) Yonsei Medical Journal, 29(4), 326-32.

[2] Masko Kusuda, Kantoriyama, Noah Kamidigo and Hideyoltakura (1998) Am. J. Trop. Med. Hg., 59(5), 801-7.

[3] Rashmi Kumar T.R., Rajlakshmi T. (2008) J. of Cytology, 25(1), 10-12.

[4] Das D.K., Francis J.M., Sharma P.N., John B., George S.S., Mallik M.K., et al. (2009) Diagn. Cytopathol., 37(8), 564-73.

[5] Juan Rosai (2004) Rosai \& Ackerman's Surgical Pathology, Ninth Edition, II, 1877-2018.

[6] Stephen Sternberg (1999) Diagnostic Surgical Pathology, Third Edition, II, 709-778.

[7] Chhieng D.C., Cangiarella J.F., Symmans W.F., Cohen J.M. (2001) Cancer Cytopathol., 93, 52-9.

[8] Mathur S. and Verma K. (2005) ActaCytol., 49, 373-6.

[9] Khurana K.K., Stanley M.W., Powers C.N., Pitman M.B. (1998) Cancer, 84, 84-91.

[10]Romangnani S., Ferrini P.L.R. and Ricci M.(1985) Semin Hematol., 22,41.

[11]Chawla P.L., Stutzmsn L., Dubois R.E., Kim U. and Sokal J.E. (1970) Am. J. Med., 48,85. 